\title{
Integer filling factor phases and isospin in vertical diatomic artificial molecules
}

\author{
D. G. Austing,,${ }^{1,2}$ S. Tarucha,${ }^{1,3}$ H. Tamura, ${ }^{1}$ K. Muraki, ${ }^{1}$ F. Ancilotto, ${ }^{4}$ M. Barranco,${ }^{5}$ \\ A. Emperador, ${ }^{5}$ R. Mayol, ${ }^{5}$ and M. $\mathrm{Pi}^{5}$ \\ ${ }^{1}$ NTT Basic Research Laboratories, NTT Corporation, 3-1 Morinosato Wakamiya, Atsugi, Kanagawa, 243-0198, Japan \\ ${ }^{2}$ Institute for Microstructural Sciences M23A, National Research Council of Canada, Ottawa, Ontario K1A OR6, Canada \\ ${ }^{3}$ Department of Physics and ERATO Mesoscopic Correlation Project, University of Tokyo, 7-3-1 Hongo, Bunkyo-ku, \\ Tokyo, 113-0033, Japan \\ ${ }^{4}$ INFM (Udr Padova and DEMOCRITOS National Simulation Center, Trieste, Italy) and Dipartimento di Fisica "G. Galilei", \\ Università di Padova, via Marzolo 8, I-35131 Padova, Italy \\ ${ }^{5}$ Departament ECM, Facultat de Física, Universitat de Barcelona, E-08028 Barcelona, Spain
}

(Received 11 March 2004; published 30 July 2004)

\begin{abstract}
Integer filling factor phases of many-electron vertically coupled diatomic artificial quantum dot molecules are investigated for different values of the interdot coupling. The experimental results are analyzed within local-spin density functional theory for which we have determined a simple lateral confining potential law that can be scaled for the different coupling regimes, and Hartree-Fock theory. Maximum density droplets composed of electrons in both bonding and antibonding or just bonding states are revealed, and interesting isospinflip physics appears for weak interdot coupling when the systematic depopulation of antibonding states leads to changes in isospin.
\end{abstract}

DOI: $10.1103 /$ PhysRevB.70.045324

PACS number(s): 73.21.La, 36.40.Ei, 71.15.Mb

\section{INTRODUCTION}

Semiconductor quantum dots (QD's) are regarded as artificial atoms whose physical properties can be easily tailored to a large extent by changing external parameters like the confining electrostatic potential, and by applying readily attainable static magnetic fields. ${ }^{1}$ High quality disk-shaped vertical QD's containing a tunable number of electrons starting from zero have revealed a wealth of atomiclike properties. ${ }^{2}$

One of the most appealing analogies with natural atoms is the capability of forming molecules. Artificial quantum molecules (QM's) composed of two vertically coupled semiconductor QD's are the subject of much experimental and theoretical investigation. ${ }^{3-15}$ In this paper we discuss integer filling factor phases that appear in vertical QM's that have different values for the thickness $b$ of the central barrier separating the two QD's when a magnetic field $\left(B_{\|}\right)$is applied parallel to the drain current $I_{d}$ flowing through the two QD's (see schematic of the submicron circular mesa in the Fig. 1 inset). We also investigate "isospin," a useful quantum number (effectively the bond order in molecular physics ${ }^{16,17}$ ), and magnetic field induced transitions in isospin. Our transistor devices incorporating the vertical QM's ${ }^{4}$ are well suited to observe changes in the molecular ground state (GS) configurations as a function of magnetic field.

This paper is organized as follows. In Sec. II, we describe the quantum molecule devices and present the key experimental data (phase diagrams). In Sec. III, we give details of the principal analytical method (local-spin density-functional theory) employed to interpret the experimental data. A detailed comparison of experiment and theory (including a supplementary and relevant Hartree-Fock calculation) is then undertaken in Sec. IV. An extended discussion in Sec. V covers important issues concerning weakly coupled quantum molecules, before we summarize in Sec. VI.

\section{QUANTUM MOLECULE DEVICES AND EXPERIMENTAL PHASE DIAGRAMS}

The QM's we study are realized in a vertical geometry transistor, as illustrated schematically in Fig. 1, by placing a single gate around a submicron cylindrical mesa incorporating a GaAs $/ \mathrm{Al}_{0.2} \mathrm{Ga}_{0.8} \mathrm{As} / \mathrm{In}_{0.05} \mathrm{Ga}_{0.95} \mathrm{As}$ triple barrier structure. Full details of the starting materials, and the mesa structures are described elsewhere. ${ }^{4,14}$ The nominally identical quantum wells of these materials are of width $12 \mathrm{~nm}$, and the outer barriers are typically about 7 to $8 \mathrm{~nm}$ wide. The molecules form because the two QD's are quantum mechanically and electrostatically coupled. For the materials we typically use, the energy splitting between the bonding (B) and antibonding $(\mathrm{AB})$ sets of single particle (s.p.) Fock-Darwin states $^{1,2} \Delta_{\mathrm{SAS}}$, one of the key parameters that determine the electronic properties of the QM, can be varied from $\sim 3.5 \mathrm{meV}$ for $b=2.5 \mathrm{~nm}$ (strong coupling) to $\sim 0.3 \mathrm{meV}$ for $b=6.0 \mathrm{~nm}$ (weak coupling). ${ }^{4}$ Each dot in the molecule can be pictured as a circular disk of thickness $\sim 10 \mathrm{~nm}$, and with an effective diameter $(\sim 100 \mathrm{~nm}$ in the few-electron limit $)$ determined by the depletion region spreading from the sidewall of the mesa, whose extent is regulated by the action of the Schottky gate. Strong (weak) quantum mechanical coupling means $\Delta_{\mathrm{SAS}} \gtrsim \hbar \omega_{0}\left(\Delta_{S A S} \ll \hbar \omega_{0}\right)$, where $\hbar \omega_{0}$ is the strength of the lateral radial harmonic oscillator potential (see Sec. III for an extended discussion of the values we use for this important quantity). If the coupling is very strong, the QM should behave like a single QD in the few-electron limit where only B states are (initially at least) populated. ${ }^{14}$ In contrast, for weak coupling, the electrostatic coupling is dominant, and the QM takes on the characteristics of two practically separate QD's. ${ }^{14}$ If the constituent dots of the QM are identical, the B and AB s.p. Fock-Darwin states would be shared 50-50\% between the two dots. In this case, the B (AB) states are truly symmetric (antisymmetric) states. 


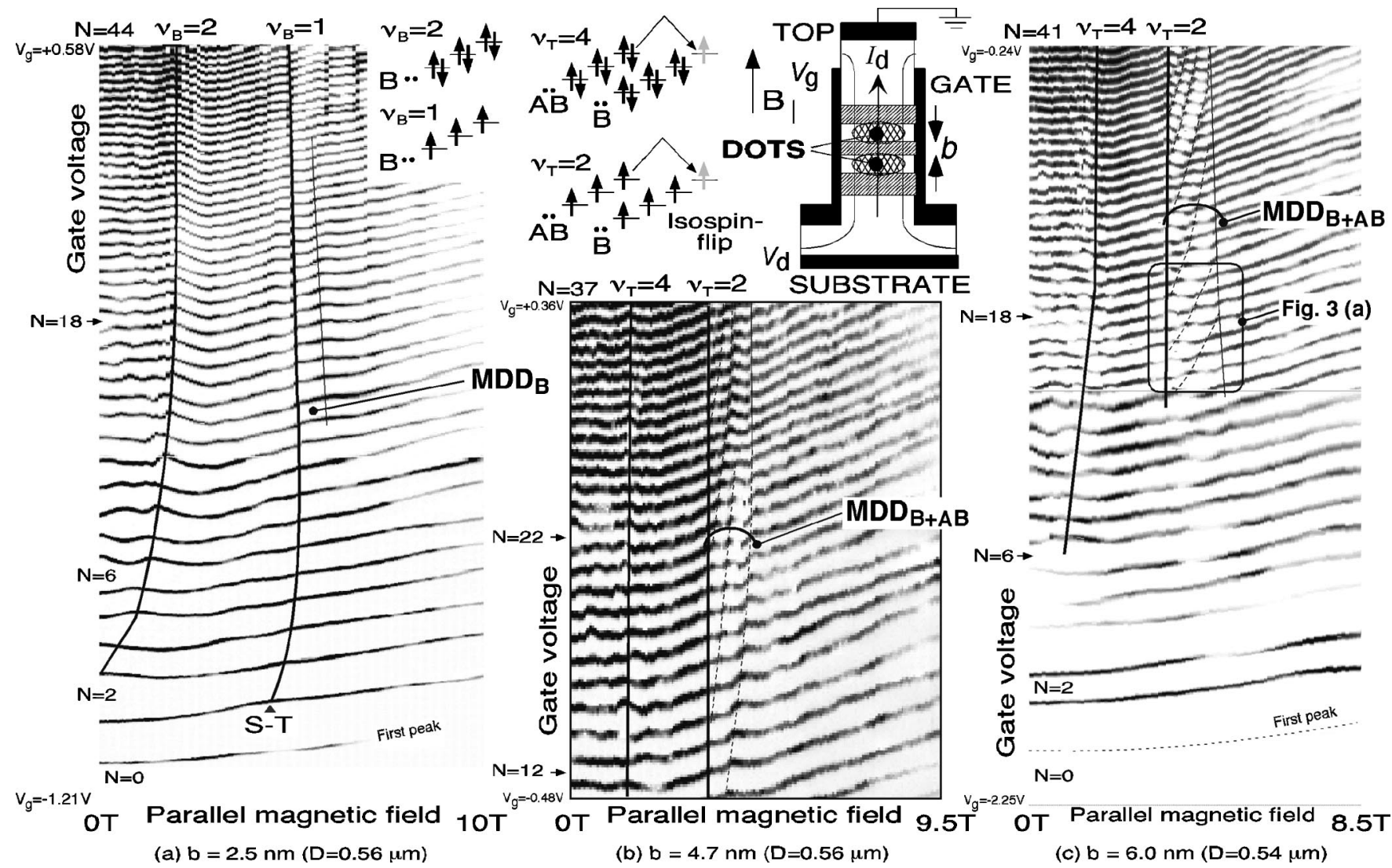

FIG. 1. Experimental $B_{\|}-N$ phase diagrams for QM structures with (a) $b=2.5 \mathrm{~nm}$, (b) $b=4.7 \mathrm{~nm}$, and (c) $b=6.0 \mathrm{~nm}$. The diagrams actually show Coulomb oscillation peaks in the $B_{\|}-V_{\mathrm{g}}$ plane. The first peak is often weak, and is indicated by a dotted line in (c). The variations in amplitude (arbitrary scale) of the current peaks depend strongly on $N, B_{\|}$, and $b$, but are not discussed here. Insets show schematic of vertical QM device, and cartoons of the arrangement of electrons in B and AB s.p. states, and isospin-flips, for certain integer filling factors.

The two vertically coupled QD's are located inside circular mesas of geometric diameter $D<1 \mu \mathrm{m}$. Drain current $I_{d}$ flows through the two QD's in response to a bias voltage $V_{d}$ applied between the substrate contact and grounded top contact, and voltage on the single surrounding side gate $V_{g}$. Attributes of the QM discussed in this paper are identified by measuring the properties of the current (Coulomb) oscillations on applying a magnetic field at temperature $\sim 100 \mathrm{mK}^{2}$.

Figure 1 shows the $B_{\|}-N$ phase diagrams for (a) $b$ $=2.5 \mathrm{~nm}$, (b) $b=4.7 \mathrm{~nm}$, and (c) $b=6.0 \mathrm{~nm}$ QM's. The electron number $N$ extends up to $\sim 40$. The diagrams actually show the $B_{\|}$evolution of the Coulomb oscillations (effectively the GS electrochemical potentials) observed on sweeping the gate voltage in the presence of a small bias $\left(V_{d}\right.$ $\sim 0.2 \mathrm{mV}$ ).

The $b=2.5 \mathrm{~nm}$ QM phase diagram has striking similarities to that of a single QD, ${ }^{18}$ so by studying the pattern of "wiggles" for each Coulomb oscillation, we can identify two threshold lines marking the onset of integer filling factor $\nu$, phases (regions) labeled $\nu_{\mathrm{B}}=2$ and $\nu_{\mathrm{B}}=1$. The former essentially marks the end of the s.p. Fock-Darwin level crossings, and the latter identifies the start of the spin-polarized compact maximum density droplet (MDD) phase. ${ }^{19}$ Here $\Delta_{\text {SAS }}$ is sufficiently large that hardly any higher-lying $\mathrm{AB}$ states play a role, so this QM clearly behaves like a single QD. ${ }^{20}$ The $\nu_{\mathrm{B}}=1$ line originates from the $N=2$ spin singlet-triplet (S-T) transition kink. ${ }^{6}$ Also, before (after) the MDD region, labeled $\mathrm{MDD}_{\mathrm{B}}$ because only the B s.p. states are occupied, barely visible kinks (weak steps) appear and are related to spin-flips (MDD reconstructions). ${ }^{6,18}$ We point out that in addition to identifying the phases from characteristic features of the Coulomb oscillations, we can also identify the GS transitions, particularly at small $N$, by looking at the evolution with $B_{\|}$of both ground and excited states in the excitation spectra when $V_{d}$ is increased to typically $1-2 \mathrm{mV}$ (the current oscillations become current stripes). This spectroscopic technique is comprehensively described elsewhere, ${ }^{21}$ and has been applied to strongly coupled and intermediately coupled QM's for $N<7.6,11$

The phase diagrams for $b=4.7$ and $6.0 \mathrm{~nm}$ QM's are clearly different from those of the $b=2.5 \mathrm{~nm} \mathrm{QM}$ and a single QD. ${ }^{6,18,19}$ We can say the following: (i) The phase diagrams for these QM's are more complex than the phase diagram for the $b=2.5 \mathrm{~nm} \mathrm{QM}$. This is particularly so for the $b=4.7 \mathrm{~nm} \mathrm{QM}$, but is expected since it is intermediate between the strong coupling and weak coupling limits. Even for the $b=6.0 \mathrm{~nm} \mathrm{QM}$ it is not readily evident how to extrapolate features in the small- $N$ region to higher $N$ (contrast this with the $\nu_{\mathrm{B}}=2$ line, and the $\nu_{\mathrm{B}}=1$ line starting from the single QD-like $N=2 \mathrm{~S}$-T kink for the $b=2.5 \mathrm{~nm} \mathrm{QM}$ ); (ii) 
Nonetheless, for $N$ exceeding $\sim 10$, we can approximately identify two lines, labeled $\nu_{\mathrm{T}}=4$ and $\nu_{\mathrm{T}}=2$ (the full meaning of these filling factor terms is given in Sec. IV). To the left (right) of $\nu_{T}=4$ each Coulomb oscillation, on the whole, oscillates weakly about a given gate voltage (shifts systematically to more positive $V_{g}$ ); (iii) To the right of $\nu_{T}=2$, there is a band (of width $\sim 1 \mathrm{~T}$ ) within which there are a series of distinct features. For consecutive current oscillations these features appear related, and we have drawn dashed lines to identify related feature. Such features are not seen for the $b=2.5 \mathrm{~nm} \mathrm{QM}$, and their origin is discussed at length in Sec. IV; and (iv). At yet higher $B_{\|}$, the dependence of the Coulomb oscillations becomes somewhat smoother again (more clearly for $b=6.0 \mathrm{~nm}$ than for $b=4.7 \mathrm{~nm}$ ). Although by no means featureless, the amplitude of the Coulomb oscillations also fall-off, as is the case for the $b=2.5 \mathrm{~nm} \mathrm{QM}$ and a single dot. ${ }^{6,18,19}$

To the best of our knowledge, no attempt has previously been made to explain even global features of the vertical QM $B_{\|}-N$ phase diagrams over a wide range of $N$ and $b$. While the concept of isospin has often been discussed in the literature, we found that hardly any published model comes close to adequately accounting for the observed properties of our particular real double quantum dot system as summarized in Fig. 1.

\section{MAIN ANALYTICAL METHOD: LOCAL-SPIN DENSITY-FUNCTIONAL THEORY}

Our principle interpretation of Fig. 1 is based on localspin density-functional theory (LSDFT) following methods comprehensively described in Refs. 13-15, 22, and 23. Finite thickness effects are included, and axial symmetry is assumed. A relaxation method is employed to solve the partial differential equations arising from a high order discretization of the Kohn-Sham equations on a spatial mesh in cylindrical coordinates. The exchange-correlation energy has been taken from Perdew and Zunger. ${ }^{22}$ We have used values appropriate for GaAs for the effective gyromagnetic factor $g_{s}^{*}$, dielectric constant $\epsilon$, and electron effective mass $m^{*}$ (in units of the bare electron mass $m_{e}, m=m^{*} m_{e}$ ): specifically, $g_{s}^{*}=-0.44, \epsilon$ $=12.4$, and $m^{*}=0.067$ (see Refs. 13 and 15 for full details). To span a large range of $N$ and reduce computational effort, only QM's with a number of electrons $N=4 M$ with $M=3$ to 9 have been comprehensively analyzed (although we did check the LSDFT at some other $N$ values for $b=6.0 \mathrm{~nm}$ as discussed in Sec. IV). The accuracy of the LSDFT for the $B_{\|}$ values of interest has been assessed ${ }^{15}$ by comparing single QD phase diagrams obtained by LSDFT and current spindensity functional theory, ${ }^{24}$ and also with some exact QM results. ${ }^{10,11}$

The QM is specifically modeled by stacking two equal QD's in the direction parallel to $I_{d}$ (equivalently $B_{\|}$). Along this (vertical) symmetry axis, the QM is confined by two identical quantum wells of width $12 \mathrm{~nm}$ and depth $225 \mathrm{meV}$, and separated by a distance $b$. We first assume the two quantum wells are identical, but consider in Sec. V, if by treating the two quantum wells as being slightly disimilar (mismatched), we can stabilize one particular phase $\left(\mathrm{MDD}_{\mathrm{B}}\right)$ in the weak coupling limit. Previously, we have shown that for small $N$ slightly unequal quantum wells can alter the appearance of the QM addition energy spectra at $0 \mathrm{~T}$, particularly when the coupling is weak. ${ }^{14}$ However, since in this paper we are primarily interested in the large- $N$ QM phase diagrams, and the influence of any small asymmetry is observed to decrease with $N$ even for weakly coupled QM's, ${ }^{5}$ we start with a symmetric double quantum well potential for the vertical direction in the LSDFT calculation. In the lateral direction, the QM is confined by a radial symmetric harmonic oscillator potential $m \omega_{0}^{2} r^{2} / 2$ of strength $\hbar \omega_{0}$.

The choice for the value of $\hbar \omega_{0}$ is clearly crucial to achieve a quantitative description of the QM phases. This can be easily seen from the expression for the s.p. energies in the noninteracting electron model at $B_{\|}=0 \mathrm{~T}$, namely, for the set of $\mathrm{B}$ or $\mathrm{AB}$ Fock-Darwin states, $\varepsilon=\hbar \omega_{0}[2 n+|l|+1]+\varepsilon_{z}$, where $\varepsilon_{z}$ is the energy of the lowest $\mathrm{B}$ or $\mathrm{AB}$ state of the double quantum well, $n$ is the s.p. radial quantum number, and $l$ is the s.p. orbital angular momentum quantum number (due to the convention commonly adopted for the s.p. wave functions, ${ }^{13}$ the s.p. orbital angular momentum is actually $-l$ ), with $l=0, \pm 1, \pm 2, \ldots$. We note that for an axially symmetric system, only the projection of the orbital angular momentum on the symmetry axis has a meaning. In a simple picture, for a sufficiently large value of $\Delta_{\mathrm{SAS}}\left(>\hbar \omega_{0}\right)$, electrons, initially at least, fill just B s.p. states, in a manner like that for a single QD. However, for $\Delta_{\mathrm{SAS}}<\hbar \omega_{0}$, the QM can easily minimize its energy by populating some $\mathrm{AB}$ s.p. states. Thus, the value of $\Delta_{\text {SAS }}$ relative to $\hbar \omega_{0}$ will have a dramatic effect on the electronic properties of QM's . ${ }^{7-10,12-14}$

For small- $N$ values, $\hbar \omega_{0}$ is often taken to be $N$-independent. We ourselves have indeed previously employed such a $N$-independent $\hbar \omega_{0}$ in such cases. ${ }^{8,14,15} \mathrm{How}$ ever, realistically $\hbar \omega_{0}$ does depend on $N .^{6,11,25}$ For large- $N$ values of interest in this paper, an $N$-dependent $\hbar \omega_{0}$ is justifiable. ${ }^{6}$ Retaining the simplicity of the harmonic oscillator model, the effective strength of the lateral confinement energy we employ in the LSDFT calculations to explain the large- $N$ QM data here, and include screening, as simply and realistically as possible, ${ }^{6}$ is of the form $\hbar \omega_{0}=\kappa / N^{1 / 4}$, where $\kappa$ is an adjustable parameter (how we determine its value is discussed in Sec. IV). Defining $N_{\mathrm{B}}\left(N_{\mathrm{AB}}\right)$ as the number of electrons in the $\mathrm{B}(\mathrm{AB})$ states, and the isospin quantum number ${ }^{17}$ as $I_{z}=\left(N_{\mathrm{B}}-N_{\mathrm{AB}}\right) / 2$, we have generalized this expression to

$$
\hbar \omega_{0}=\frac{\kappa}{N_{\mathrm{B}}^{1 / 4}}=\frac{\kappa}{\left(I_{z}+N / 2\right)^{1 / 4}} .
$$

The rationale is simple. In the strong coupling limit, the QM behaves like a single $\mathrm{QD}$, and $N_{\mathrm{B}}=N$, so we recover the usual expression. In the weak coupling limit, one can regard each constituent quantum dot as hosting half the total number of electrons of the QM, i.e., $N_{\mathrm{B}}=N_{\mathrm{AB}}=N / 2$, and so the lateral confinement strength is fixed by $N / 2$ and not $N$. For intermediate coupling, where $N_{\mathrm{B}}$ is not known a priori, we "guess" its value at $B_{\|}=0 \mathrm{~T}$ and iterate until the actual $N_{\mathrm{B}}$ value is obtained. For one single $\mathrm{QD}$, it is worth mentioning that some successful attempts have recently been made to 


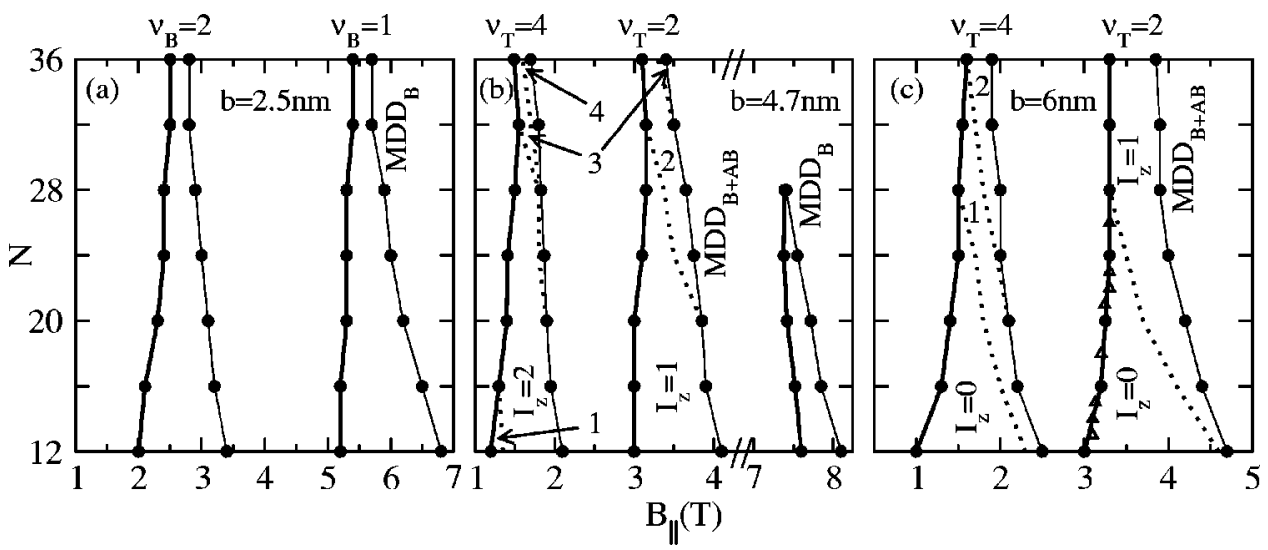

FIG. 2. $B_{\|}-N$ phase diagrams calculated by LSDFT for QM structures with (a) $b=2.5 \mathrm{~nm}$, (b) $b=4.7 \mathrm{~nm}$, and (c) $b=6.0 \mathrm{~nm}$. The low (high) field boundary of each integer filling factor phase of finite width is drawn bold (feint). Only values corresponding to $N$ $=12,16, \ldots, 32,36$ are meaningful here [except for extra points in (c) on the left boundary of the MDD $_{\mathrm{B}+\mathrm{AB}}$ phase as discussed in the text]. Within certain phases, regions of different isospin are separated by dotted lines. The $B_{\|}$axis is broken in (b).

obtain the confining potential from the electrostatics of "model" devices with the aim of avoiding the more phenomenological point of view we adopt here. ${ }^{26}$ However, there is still arbitrariness and uncertainty even in these calculations for single quantum dots, because to the best of our knowledge, no model yet exists to accurately include deviations from ideality and randomness which occur in all real quantum dot structures. ${ }^{27}$ To the best of our knowledge, fullblown self-consistent models of large- $N$ vertical QM's in a magnetic field have not yet been reported.

\section{DETAILED COMPARISON OF EXPERIMENT AND THEORY}

For the strongly coupled QM, from Fig. 1(a), taking $\kappa$ $=6.91 \mathrm{meV}$, the experimental low $-B_{\|}$border of the $\mathrm{MDD}_{\mathrm{B}}$ phase is well reproduced. We choose this border for the fit because it is well defined (and then use the same $\kappa$ for the more weakly coupled QM's). The corresponding calculated large- $N$ phase diagram is displayed in Fig. 2(a). In the LSDFT calculations here, at $B_{\|}=0 \mathrm{~T}$, electrons occupy only B s.p. states, at least up to $N=36$, confirming the viewpoint that this QM behaves like a single QD. ${ }^{20}$ The $\mathrm{MDD}_{\mathrm{B}}$ consists of same spin electrons occupying just B s.p. states, such that the total orbital angular momentum $L_{z}=N(N-1) / 2$, and corresponds to total filling factor $\nu_{T}=\nu_{\mathrm{B}}=1 .{ }^{19}$ An experimental feature this calculation, and indeed other calculations Refs. 9,10 , and 12 , is unable to reproduce well, is the $B_{\|}$range of stability, at large $N$, of the $\mathrm{MDD}_{\mathrm{B}}$ compared to that of the MDD for a single QD. ${ }^{6}$ Experimentally, the former is $\sim 40 \%$ narrower than the latter, whereas the LSDFT yields a similar range for both. The calculation also reveals the $\nu_{T}=\nu_{\mathrm{B}}=2$ phase (spin unpolarized for even- $N$ ), again made of just $\mathrm{B}$ s.p. states. The low- $B_{\|}$border of this phase agrees well with the distinct $\nu_{\mathrm{B}}=2$ line in the experiment, but the high- $B_{\|}$ border, identifying the first spin-flip, is not clear in the data shown, as is also the case for a single vertical QD. ${ }^{18}$

As discussed in Sec. II, the phase diagrams noticeably change when the coupling is reduced, and as far as we know, no model exists to explain all the experimental observations.
We focus first, in this Sec., on some of the more prominent features, and in Sec. V we address other important issues. In the weak coupling limit, $N_{\mathrm{B}} \sim N_{\mathrm{AB}}$ at $B_{\|}=0 \mathrm{~T}$. As the field is applied, two cohabiting MDD's can develop. One is made of B s.p. states $\left(\mathrm{MDD}_{\mathrm{B}}\right)$, and the other is made of $\mathrm{AB}$ s.p. states $\left(\mathrm{MDD}_{\mathrm{AB}}\right)$. We call this phase $\mathrm{MDD}_{\mathrm{B}+\mathrm{AB}}$, and $\nu_{T}=2\left(\nu_{\mathrm{B}}=1\right.$ plus $\left.\nu_{\mathrm{AB}}=1\right)$. By inspection, we can identify the onset of this phase at sufficiently large $N$ in Fig. 1(c) and less clearly in Fig. 1(b). The onset calculated by LSDFT of the $\mathrm{MDD}_{\mathrm{B}+\mathrm{AB}}$ phase in Figs. 2 is within $\sim 15 \%$ (on the low side) of the marked onsets in Fig. 1. We emphasize that we used the same $\kappa$ value as for the $b=2.5 \mathrm{~nm} \mathrm{QM}$, and had we used the regular $\kappa / N^{1 / 4}$ law instead of the $\kappa / N_{\mathrm{B}}^{1 / 4}$ law, the calculated $\mathrm{MDD}_{\mathrm{B}+\mathrm{AB}}$ phase would have been shifted to still lower $B_{\|}$by $\sim 0.7 \mathrm{~T}$. Lacking any better model, we have thus satisfactorily reproduced the onset of the $\mathrm{MDD}_{\mathrm{B}+\mathrm{AB}}$.

In the LSDFT calculations for $N=4 M$, the electrons in the $\mathrm{MDD}_{\mathrm{B}}$ and $\mathrm{MDD}_{\mathrm{AB}}$ constituting the $\mathrm{MDD}_{\mathrm{B}+\mathrm{AB}}$ phase in the $b=4.7$ and $6.0 \mathrm{~nm}$ QM's initially have s.p. orbital angular momentum values from 0 to $\sim N / 2$. As $N$ or $B_{\|}$increase, there is a clear tendency for $N_{\mathrm{B}}$ and $N_{\mathrm{AB}}$ to become unbalanced with $N_{\mathrm{B}}\left(N_{\mathrm{AB}}\right)$ increasing (decreasing), so in the $\mathrm{MDD}_{\mathrm{B}+\mathrm{AB}}$ phase the $\mathrm{QM}$ can undergo isospin transitions (isospin-flips). Isospin-flips can occur at weaker fields, as we discuss in Sec. V, but they are then easily masked by s.p. level crossings and spin-flips, ${ }^{12}$ i.e., isospin-flips should be most visible within the $\mathrm{MDD}_{\mathrm{B}+\mathrm{AB}}$ phase. There is only one (dotted) $\Delta I_{z}=+1$ transition line for the $N$-values shown in Fig. 2 for the $b=6.0 \mathrm{~nm} \mathrm{QM}$, and two such lines for the $b$ $=4.7 \mathrm{~nm} \mathrm{QM}\left(\Delta_{\mathrm{SAS}}\right.$ is smaller in the former than the latter so $\mathrm{AB}$ states are "harder" to depopulate). Also, the $\mathrm{MDD}_{\mathrm{B}+\mathrm{AB}}$ apparently reconstructs to some lower density droplet at higher $B_{\|}$well before $I_{z}$ has reached the maximum possible value of $N / 2$. In the experimental data, the right boundary of the $\mathrm{MDD}_{\mathrm{B}+\mathrm{AB}}$ phase in Figs. 1(b) and 1(c) is indeed not very distinct, but beyond the left onset of this phase, for $N>10$, there are clearly a series of features (connected by dashed lines) that originate from the $\nu_{\mathrm{T}}=2$ line that have a characteristic with $N$ or $B_{\|}$not seen in any features in the $b$ $=2.5 \mathrm{~nm} \mathrm{QM}$, or in a single QD. ${ }^{18} \mathrm{We}$ attribute these to isospin-flips. 

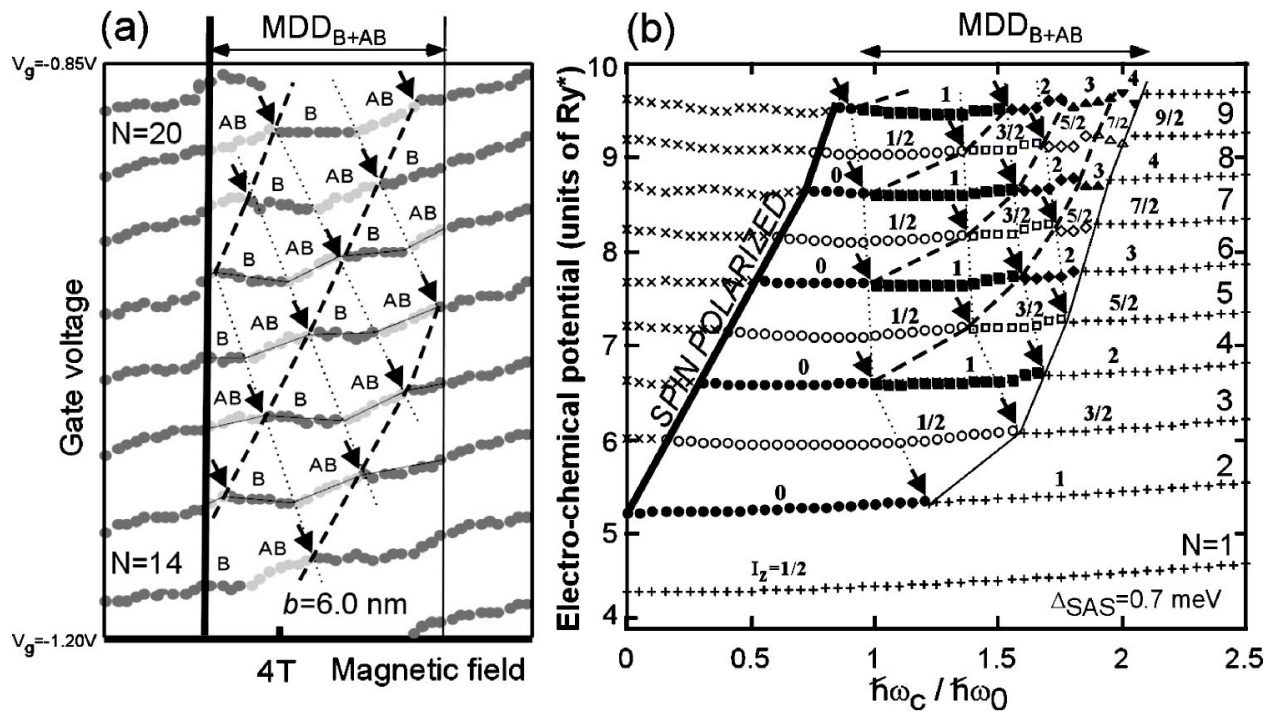

FIG. 3. (a) Expanded view of phase diagram taken from Fig. 1(c) for the $b=6.0 \mathrm{~nm}$ QM in the vicinity of the MDD $\mathrm{B}_{\mathrm{AB}}$ for $13<N<21$. (b) Evolution of the GS electrochemical potentials (in units of the effective Rydberg constant, Ry ${ }^{*}=5.93$ meV) with magnetic field (in units of the cyclotron energy $\hbar \omega_{c}$ normalized by the constant lateral confinement energy $\hbar \omega_{0}=3 \mathrm{meV}$ ) obtained by an unrestricted HF calculation for a QM with $\Delta_{\mathrm{SAS}}=0.7 \mathrm{meV}$ for $N<10$ showing regions of different $I_{z}$ within the $\mathrm{MDD}_{\mathrm{B}+\mathrm{AB}}$. In (a), feint guide lines are drawn on four consecutive peaks in the $\mathrm{MDD}_{\mathrm{B}+\mathrm{AB}}$ region to emphasise the underlying "honey-comb" like structure. Within this region, the dotted and dashed lines, and the arrows, have the same meaning in (a) and (b) as discussed in the text.

We now explain why the dotted $\Delta I_{z}=+1$ transition lines in the $\mathrm{MDD}_{\mathrm{B}+\mathrm{AB}}$ region of the more weakly coupled QM's calculated by LSDFT should have the opposite trend, with $B_{\|}$, to the dashed lines joining the observed isospin-flip features in Fig. 1. Figure 3 shows (a) an expanded view of part of the experimental data taken from Fig. 1(c) for the $b$ $=6.0 \mathrm{~nm} \mathrm{QM}$, and (b) the results of a relevant Hartree-Fock (HF) model calculation showing regions of different $I_{z}$ within the $\mathrm{MDD}_{\mathrm{B}+\mathrm{AB}}$ that complements the phase diagrams in Figs. 2(b) and 2(c) calculated by LSDFT.

Full details of the unrestricted self-consistent HF implementation can be found in Ref. 8. The calculation shows the B-field evolution of the GS electrochemical potentials. $\Delta_{\text {SAS }}$ is set to $0.7 \mathrm{meV}$. The calculation is only for (consecutive) $N$-values from 1 to 10 , and $\hbar \omega_{0}$ is a constant with a value of $3 \mathrm{meV}$. Thus, the region of $N$ is below that of the main focus of Figs. 1 and 2, and since the lateral confinement energy is $\mathrm{N}$-independent, we do not expect this calculation to reproduce well the position of the integer filling factor phase boundaries. Additionally, correlation effects are not properly included, so one should be cautious when the spin is not maximal, for example, in spin-flip regions below the $\mathrm{MDD}_{\mathrm{B}+\mathrm{AB}}$. Nonetheless, it does shed light on the general properties of the spin-polarized $\mathrm{MDD}_{\mathrm{B}+\mathrm{AB}}$ region. We can say the following which clearly extrapolate to higher $N$ : (i) To the right of the line marked "spin polarized" the $\mathrm{MDD}_{\mathrm{B}+\mathrm{AB}}$ exists, and on increasing the magnetic field $\mathrm{AB}$ states depopulate one-by-one until they are all empty. Each isospin-flip is marked by an arrow; (ii) For even- $N$ (odd- $N$ ), there are changes in $I_{z}$ with $\Delta I_{z}=+1$ such that $I_{z}$ follows the sequence $0,1,2, \ldots, N / 2(1 / 2,3 / 2, \ldots, N / 2)$; (iii) For even$N$ values or odd- $N$ values separately, regions of different $I_{z}$ are separated by dotted lines which run approximately from bottom-right to top-left (this is also consistent with what is shown in Figs. 2(b) and 2(c) for even- $N$ values $12,16, \ldots, 32,36$ ); (iv) For consecutive- $N$ values (even and odd), the removal of an electron from a specific AB-state is identified by a dashed line that runs from bottom-left to topright. There are several of these lines, since as $N$ increases, $\sim N / 2$ AB states need to be depopulated. Such lines we expect to see in the experimental data and indeed we do see these (dashed) lines in Figs. 1(b) and 1(c); and (v) As $N$ increases, for even- $N$, the extent of the $I_{z}=0$ region decreases steadily to zero since the $I_{z}=0 \rightarrow 1$ transition line terminates on the "spin polarized" line. This trend seems likely to occur for other (both even- and odd- $N$ ) $I_{z}$ regions, and this would be consistent with the LSDFT calculations.

The experimental data is for the $b=6.0 \mathrm{~nm}$ QM and focuses on the boxed region of Fig. 1(c) near $4 \mathrm{~T}$ showing the Coulomb oscillations for $N$-values between 14 and 20 . We can say the following: (i) Starting from the left boundary of the $\mathrm{MDD}_{\mathrm{B}+\mathrm{AB}}$ region, there are "wiggles," both peaks and troughs, in the Coulomb oscillation positions which seem not to clearly continue beyond the right boundary. Actually these "wiggles" too are (weakly) present in the GS electrochemical potentials in the HF calculation; (ii) For any given Coulomb oscillation, we attribute each peak marked by an arrow to the magnetic field induced transfer of an electron from a specific $\mathrm{AB}$ state to an empty B state (i.e., an isospin-flip: also see relevant cartoon in Fig. 1). In a s.p. picture, to the left (right) of each such peak, the electron most recently added to the QM is placed in the highest unoccupied "up-going" $\mathrm{AB}$ ("down-going" B) state. To make this clear in the figure, the "up-going" ("down-going") part of each Coulomb oscillation within the $\mathrm{MDD}_{\mathrm{B}+\mathrm{AB}}$ region is artificially colored light-grey (dark-grey). Alternatively, we have also drawn faint guide lines on four consecutive Coulomb oscillations in the $\mathrm{MDD}_{\mathrm{B}+\mathrm{AB}}$ region to emphasize the underlying "honey- 
comb" like structure arising from the pattern of alternately filling $\mathrm{AB}$ and $\mathrm{B}$ states. Clearly the number of (arrowed) peaks is much less than $N / 2$, so apparently the $\mathrm{MDD}_{\mathrm{B}+\mathrm{AB}}$ in reality breaks down well before $I_{z}$ reaches $N / 2$ (again consistent with the LSDFT calculation); (iii) For consecutive- $N$ values (even and odd), the (arrowed) peaks marking the depopulation of a given $\mathrm{AB}$ state lie on a dashed line. There is in fact a one-to-one correspondance with the three dashed lines here in (a) with those in the boxed region of Fig. 1(c). Such lines run from bottom-left to top-right, and they also correspond to the dashed lines with the same trend in the HF calculation; and (iv) Dotted lines, alternately linking peaks and troughs in neighboring Coulomb oscillations run from bottom-right to top-left and terminate on the left boundary of the $\mathrm{MDD}_{\mathrm{B}+\mathrm{AB}}$. These lines identify the boundaries of regions that for even- $N$ and odd- $N$ values separately have the same $I_{z}$ number (again consistent with both the HF and LSDFT calculations). For any given Coulomb oscillation, the highest unoccupied $\mathrm{B}$ and $\mathrm{AB}$ states are alternately filled between the dotted lines.

We make some further comments: (i) No "exact" calculation exists for the QM physics we focus on, and both HF theory and LSDFT are based on approximations. Nonetheless, the HF and LSDFT calculations we present are qualitatively consistent with the experimental data for $\mathrm{MDD}_{\mathrm{B}+\mathrm{AB}}$, although the HF calculation clearly overestimates the number of observed isospin-flips, while the LSDFT slightly underestimates the number of observed isospin-flips $(\ll N / 2)$. Quantitatively, the LSDFT with an $N$-dependent $\hbar \omega_{0}$ also explains best the position and extent of the integer filling factor phases; (ii) Just in case our choice of calculating the phase diagrams in Fig. 2 by LSDFT for just the even- $N$ values of $12,16, \ldots, 32,36$ did not capture the true general picture, we additionally determined the position of the left boundary of the $\mathrm{MDD}_{\mathrm{B}+\mathrm{AB}}$, and the initial value of $I_{z}$ on and just to the right of this boundary for other $N$ values (both even and odd), specifically for the $b=6.0 \mathrm{~nm}$ QM. For $N$ $=14,18,22$, and $26(N=13,15,21,23)$, as expected, and consistent with the HF picture, $I_{z}=0\left(I_{z}=1 / 2\right)$, and the left boundary of $\mathrm{MDD}_{\mathrm{B}+\mathrm{AB}}$ at these $N$ values is always within $0.1 \mathrm{~T}$ of the extrapolated left boundary line in Fig. 2(c). These "extra" data points have been added to this figure (triangles).

\section{V. $\nu_{T}=4$ PHASE AND MDD ${ }_{\mathrm{B}}$ PHASE FOR WEAKLY COUPLED QUANTUM MOLECULES}

We conclude by discussing two important topics, addressed directly by the LSDFT calculations, for the weakly coupled QM's: namely, the isospin properties within the adjacent integer filling factor phase below the $\mathrm{MDD}_{\mathrm{B}+\mathrm{AB}}$ phase $\left(\nu_{T}=4\right)$, and the stability of the $\operatorname{MDD}_{\mathrm{B}}$ phase $\left(\nu_{T}=1\right)$ located beyond the $\mathrm{MDD}_{\mathrm{B}+\mathrm{AB}}$ phase.

For the $b=4.7$ and $6.0 \mathrm{~nm}$ QM's, the LSDFT calculations reveal a $\nu_{T}=4$ phase built from the $\nu_{\mathrm{B}}=2$ and $\nu_{\mathrm{AB}}=2$ phases. In the experiment, we can currently only clearly identify the start of this phase at a $B_{\|}$of about half that of the onset of the $\mathrm{MDD}_{\mathrm{B}+\mathrm{AB}}$ phase. Note that our original choice of $N=4 M$ was partly motivated by the idea that the $\nu_{T}=4$ phase would
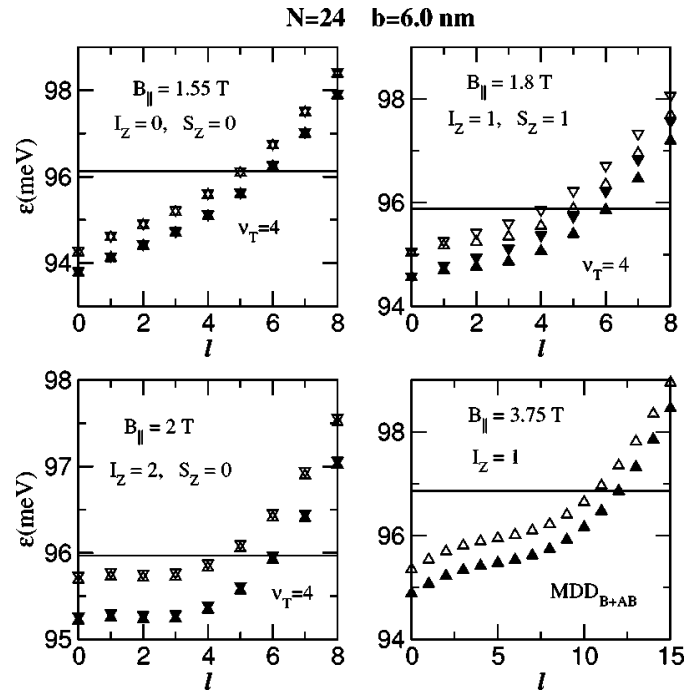

FIG. 4. Single particle energies $\varepsilon$ for the $N=24 b=6.0 \mathrm{~nm}$ QM as a function of the s.p. orbital angular momentum $l$. Boldfaced (open) triangles correspond to B (AB) states. Upward (downward) triangles represent $\uparrow(\downarrow)$ spin electrons. The horizontal line indicates the Fermi level. The $B_{\|}, S_{z}$ and $I_{z}$ values are indicated in each panel.

correspond to total spin $S_{z}=0$ states as for single QD's. ${ }^{28}$ However, due to the isospin degree of freedom, this is not always the case, as can be seen in the rightmost panel of Fig. 2 for instance, where a "strip" of $S_{z}=1\left(I_{z}=1\right)$ states splits the $S_{z}=0\left(I_{z}=0,2\right)$ states of the $\nu_{T}=4$ phase. More generally, for $N=4 M$, the LSDFT gives alternating regions of $S_{z}$ $=1\left(I_{z}=\right.$ odd $)$ and $S_{z}=0\left(I_{z}=\right.$ even $)$ states that are separated by dotted lines in Fig. 2. The value $S_{z}=1$ arises because there are two unpaired electrons, one each in edge $\mathrm{B}$ and $\mathrm{AB}$ s.p. states (see $\nu_{T}=4$ schematic inset in Fig. 1). Defining the $\nu_{T}$ $=4$ phase in a broader sense, as the last (nearly) $S_{z}=0$ phase with occupied s.p. states whose orbital angular momenta range from $l=0$ to $\sim N / 4$ in the four $\mathrm{B}(\uparrow, \downarrow)$ and $\mathrm{AB}(\uparrow, \downarrow)$ sub-bands, one may picture a "quasi" $\nu_{T}=4$ phase with a rich internal isospin structure for which, as a general rule, the constraint $N=4 M$ implies that odd isospin regions also correspond to $S_{z}=1$ values. The even-to-odd isospin transitions in the $\nu_{T}=4$ phase for weakly coupled QM's are achieved simultaneously with $\Delta S_{z}=1$ jumps. Because of this, the system acquires edge magnetization, i.e., the spins of two electrons in large $l \mathrm{~B}$ and $\mathrm{AB}$ s.p. states are aligned producing a $S_{z}=1$ state, and this lifts the quasidegeneracy of $(\uparrow, \downarrow)$ B or $\mathrm{AB}$ states (recall the Zeeman energy here is very small). This is due to the spin dependence of the exchange-correlation potential. ${ }^{15,28}$ One can thus regard the isospin transistions as "spin-flip-driven." Note the odd-to-even isospin transitions proceed in the opposite way with $\Delta S_{z}=-1$ jumps. In contrast, in the $\mathrm{MDD}_{\mathrm{B}+\mathrm{AB}}$ phase, electrons are always spin polarized, i.e., the isospin transitions here are not spin-flip driven. To illustrate all these distinctions, we have displayed in Fig. 4 the $b=6.0 \mathrm{~nm}$ QM s.p. energies for $N=24$ and $B_{\|}$values corresponding to $\nu_{T}=4$, for $I_{z}=0,1$, and 2 , and also to $\nu_{T}$ $=2\left(\mathrm{MDD}_{\mathrm{B}+\mathrm{AB}}\right)$, for $I_{z}=1$.

A delicate question is whether the $\mathrm{MDD}_{\mathrm{B}}$ phase exists or not for weak coupling at higher magnetic fields. Experimen- 
tally, we cannot identify this phase at a field approximately twice above that of the $\nu_{T}=2$ line in Fig. 1. Indeed, as for the $b=2.5 \mathrm{~nm} \mathrm{QM}$ and a single QD, the amplitude of the current oscillations falls-off and other as yet unexplained features appear for $B_{\|} \geqslant 7 \mathrm{~T} .{ }^{18}$ As already noted, the experimental $b$ $=2.5 \mathrm{~nm} \mathrm{MDD} \mathrm{B}_{\mathrm{B}}$ has a narrower stability range than that of a single QD, so it appears empirically that the $\mathrm{MDD}_{\mathrm{B}}$ phase becomes less stable as $\Delta_{S A S}$ is reduced (see also Ref. 9). Turning to the calculated phases in Fig. 2, the $\mathrm{MDD}_{\mathrm{B}}$ is identified for the $b=4.7 \mathrm{~nm} \mathrm{QM}$, but the range of stability is much reduced, and it becomes "zero" for $N>28$. For the $b$ $=6.0 \mathrm{~nm} \mathrm{QM}$, if the $\mathrm{MDD}_{\mathrm{B}}$ is stable at all in any meaningful sense, it has almost zero extent, i.e., it is a "line," and is located at $\sim 8.7,8.3$, and $8.0 \mathrm{~T}$ for $N=16,20$, and 24 , respectively.

An interesting point is whether a slight energy mismatch between the two weakly coupled QD's would help to stabilize the $\mathrm{MDD}_{\mathrm{B}}$ phase. This mismatch, leading to a slightly asymmetric double dot system, is unintentionally introduced in the fabrication of the QM's. An offset of $\sim 1-2 \mathrm{meV}$ between the two quantum wells (still small relative to the depth of the two wells of $225 \mathrm{meV}$ ), has a sizeable effect on the addition energy spectra of weakly coupled "heteronuclear" QM's for $N<12 .{ }^{14}$ We wondered if mismatch might help favor the existence of the $\mathrm{MDD}_{\mathrm{B}}$ phase. However, the effects of mismatch and self-consistency, especially for the large- $N$ values here, are nontrivial. So in fact, the properties of the large $N \mathrm{MDD}_{\mathrm{B}}$ phase listed above remained practically unchanged when a realistic mismatch energy was included in the LSDFT calculation. Only for small $N$ values, i.e., when the electron interaction energy is relatively small, did we find that the mismatch helps a little to stabilize the $\mathrm{MDD}_{\mathrm{B}}$ phase. For example, a mismatch of $2 \mathrm{meV}$ yields for the $N=12, b$ $=6.0 \mathrm{~nm} \mathrm{QM}$ a narrow $\mathrm{MDD}_{\mathrm{B}}$ phase at $\sim 9.1 \mathrm{~T}$, whereas without mismatch this phase does not appear. We note that experimentally, from data published in Ref. 5, the influence of mismatch and the value of the mismatch energy diminishes steadily as $N$ increases.

\section{SUMMARY}

Gated submicron vertical triple barrier structures are ideal for studying complex and fundamental properties of coupled quantum dots, i.e., quantum dot molecules. The inter-dot distance in our QM's is determined at growth. This then allows us to study how the ground state configurations ("phases") of QM's whose constituent QD's are coupled to different degrees change as a function of applied magnetic field. At large $N$, we have clearly observed maximum density droplet states composed of electrons in both bonding and anti-bonding states (weak coupling case) or just bonding states (strong coupling case), and other integer filling factor phases. For weak interdot coupling, we have demonstrated that the existence of such phases can give rise to isospin-flip events due to the systematic one-by-one depopulation of antibonding states. Additionally, for weakly coupled quantum molecules, we have also predicted the possibility of "spin-flip-driven" isospin transistions in the $\nu_{T}=4$ phase, and discussed the reduced stability of the $\mathrm{MDD}_{\mathrm{B}}$ phase.

\section{ACKNOWLEDGMENTS}

This work is supported by the Specially Promoted Research, Grant-In-Aid for Scientific Research, by DARPAQUIST program (DAAD 19-01-1-0659), by MIUR-COFIN 2001 (Italy), and by BFM2002-01868 and 2001SGR00064 (Spain). The authors are grateful for the assistance of $\mathrm{T}$. Honda and useful discussions with K. Ono, Y. Tokura, and S. Sasaki.
${ }^{1}$ M. A. Kastner, Phys. Today 46(1), 24 (1993); R. C. Ashoori, Nature (London) 379, 413 (1996); L. Jacak, P. Hawrylak, and A. Wójs, Quantum Dots (Springer, Berlin, 1998); Mesoscopic Physics and Electronics, edited by T. Ando, Y. Arakawa, K. Furuta, S. Komiyama, and H. Nakashima (Springer, Berlin, 1998); S. M. Reimann and M. Manninen, Rev. Mod. Phys. 74, 1283 (2002).

${ }^{2}$ S. Tarucha, D. G. Austing, T. Honda, R. J. van der Hage, and L. P. Kouvenhoven, Phys. Rev. Lett. 77, 3613 (1996); L. P. Kouvenhoven, D. G. Austing, and S. Tarucha, Rep. Prog. Phys. 64, 701 (2001).

${ }^{3}$ T. Schmidt, R. J. Haug, K. V. Klitzing, A. Förster, and H. Lüth, Phys. Rev. Lett. 78, 1544 (1997); A. Lorke and R. J. Luyken, Physica B 256-258, 424 (1998); R. J. Luyken, A. Lorke, M. Fricke, J. P. Kotthaus, G. Medeiros-Ribeiro, and P. M. Petroff, Nanotechnology 10, 14 (1999); M. Bayer, P. Hawrylak, K. Hinzer, S. Fafard, M. Korkusinsky, Z. R. Wasilewski, O. Stern, and A. Forchel, Science 291, 451 (2001).

${ }^{4}$ D. G. Austing, T. Honda, Y. Tokura, and S. Tarucha, Physica B 249-251, 206 (1998).
${ }^{5}$ K. Ono, D. G. Austing, Y. Tokura, and S. Tarucha, Science 297, 1313 (2002).

${ }^{6}$ S. Amaha, D. G. Austing, Y. Tokura, K. Muraki, K. Ono, and S. Tarucha, Solid State Commun. 119, 183 (2001).

${ }^{7}$ J. J. Palacios and P. Hawrylak, Phys. Rev. B 51, 1769 (1995); J. H. Oh, K. J. Chang, G. Ihm, and S. J. Lee, ibid. 53, R13 264 (1996); H. Imamura, P. A. Maksym, and H. Aoki, ibid. 53, 12613 (1996); O. Mayrock, S. A. Mikhailov, T. Darnhofer, and U. Rössler, ibid. 56, 15760 (1997); Y. Asano, ibid. 58, 1414 (1998); Y. Tokura, D. G. Austing, and S. Tarucha, J. Phys.: Condens. Matter 11, 6023 (1999); G. Burkard, D. Loss, and D. P. DiVincenzo, Phys. Rev. B 59, 2070 (1999); G. Burkard, G. Seelig, and D. Loss, ibid. 62, 2581 (2000); L. Martín-Moreno, L. Brey, and C. Tejedor, ibid. 62, R10 633 (2000); B. Partoens and F. M. Peeters, Phys. Rev. Lett. 84, 4433 (2000); D. Sánchez, L. Brey, and G. Platero, Phys. Rev. B 64, 235304 (2001); T. Matsuse, T. Hama, H. Kaihatsu, N. Toyoda, and T. Takizawa, Eur. Phys. J. D 16, 391 (2001); S. Bednarek, T. Chwiej, J. Adamowski, and S. Szafran, Phys. Rev. B 67, 205316 (2003); N. Barberan, and J. Soto, ibid. 68, 045306 (2003); D. Jacob, B. Wunsch, and D. Pfannkuche, cond-mat/0401429 (2004). 
${ }^{8}$ H. Tamura, Physica B 249-251, 210 (1998).

${ }^{9}$ J. Hu, E. Dagoto, and A. H. MacDonald, Phys. Rev. B 54, 8616 (1996).

${ }^{10}$ M. Rontani, F. Rossi, F. Manghi, and E. Molinari, Solid State Commun. 112, 151 (1999); M. Rontani, F. Troinai, U. Hohenester, and E. Molinari, ibid. 119, 309 (2001).

${ }^{11}$ M. Rontani, S. Amaha, K. Muraki, F. Manghi, E. Molinari, S. Tarucha, and D. G. Austing, Phys. Rev. B 69, 085327 (2004).

${ }^{12}$ B. Partoens and F. M. Peeters, Physica B 298, 282 (2001); Europhys. Lett. 56, 86 (2001).

${ }^{13}$ M. Pi, A. Emperador, M. Barranco, and F. Garcias, Phys. Rev. B 63, 115316 (2001).

${ }^{14}$ M. Pi, A. Emperador, M. Barranco, F. Garcias, K. Muraki, S. Tarucha, and D. G. Austing, Phys. Rev. Lett. 87, 066801 (2001).

${ }^{15}$ F. Ancilotto, D. G. Austing, M. Barranco, R. Mayol, K. Muraki, M. Pi, S. Sasaki, and S. Tarucha, Phys. Rev. B 67, 205311 (2003).

${ }^{16}$ J. P. Dahl, Introduction to the Quantum World of Atoms and Molecules (World Sci., Singapore, 2001).

${ }^{17}$ Like other authors (Refs. 10-12 ), we take the following definition for the "isospin" quantum number: $I_{z}=\left(N_{B}-N_{A B}\right) / 2$, where $N_{B}\left(N_{A B}\right)$ is the number of electrons in the bonding (antibonding) states. We will use $I_{z}$ to label some of the QM states within certain integer filling factor phases for the more weakly coupled QM's in Secs. IV and V.

${ }^{18}$ D. G. Austing, Y. Tokura, T. Honda, S. Tarucha, M. W. S. Danoesastro, J. Janssen, T. H. Oosterkamp, and L. P. Kouwenhoven, Jpn. J. Appl. Phys., Part 1 38, 372 (1999); T. H. Oosterkamp, J. W. Jannsen, L. P. Kouwenhoven, D. G. Austing, T. Honda, and S. Tarucha, Phys. Rev. Lett. 82, 2931 (1999). We note that many features, particularly at large $N(>20)$ and large $B_{\|}(>8 \mathrm{~T})$, of the single vertical dot phase diagram have yet to be satisfactorily explained by any model.

${ }^{19}$ For MDD physics in single dots, see papers such as the following: A. H. MacDonald, S.-R. E. Yang, and M. D. Johnson, Aust. J.
Phys. 46, 345 (1993); S. M. Reimann, M. Koskinen, M. Manninen, and B. R. Mottelson, Phys. Rev. Lett. 83, 3270 (1999); S.-R. E. Yang and A. H. MacDonald, Phys. Rev. B 66, 041304(R) (2002).

${ }^{20}$ For the $b=2.5 \mathrm{~nm}$ QM device a weak feature appears in the phase diagram for $N>25$ (not resolvable in Fig. 1), just to the right of the $\nu_{\mathrm{B}}=2$ line, that can be explained by the occupation of at most a single electron in the lowest $\mathrm{AB}$ state (unpublished). The single QD like shell structure at $0 \mathrm{~T}(N<18)$ is discussed elsewhere (Refs. 6 and 14).

${ }^{21}$ L. P. Kouwenhoven, T. H. Oosterkamp, M. W. S. Danoesastro, M. Eto, D. G. Austing, T. Honda, and S. Tarucha, Science 278, 1788 (1997).

${ }^{22}$ J. P. Perdew and A. Zunger, Phys. Rev. B 23, 5048 (1981).

${ }^{23}$ M. Stopa, Phys. Rev. B 54, 13767 (1996); M. Koskinen, M. Manninen, and S. M. Reimann, Phys. Rev. Lett. 79, 1389 (1997); I. H. Lee, V. Rao, R. M. Martin, and J. P. Leburton, Phys. Rev. B 57, 9035 (1998); R. N. Barnett and U. Landman, ibid. 48, 2081 (1993); K. Hirose and N. S. Wingreen, ibid. 59, 4604 (1999); G. Onida, L. Reining, and A. Rubio, Rev. Mod. Phys. 74, 601 (2002).

${ }^{24}$ M. Ferconi and G. Vignale, Phys. Rev. B 50, 14722 (1994).

${ }^{25}$ S. Tarucha, D. G. Austing, S. Sasaki, Y. Tokura, W. van der Wiel, and L. P. Kouwenhoven, Appl. Phys. A: Mater. Sci. Process. 71, 367 (2000).

${ }^{26}$ N. A. Bruce and P. A. Maksym, Phys. Rev. B 61, 4718 (2000); S. Bednarek, B. Szafran, and J. Adamowski, ibid. 64, 195303 (2001); P. Matagne and J. P. Leburton, ibid. 65, 235323 (2002); S. Bednarek, B. Szafran, K. Lis, and J. Adamowski, ibid. 68, 155333 (2003).

${ }^{27}$ P. Matagne, J. P. Leburton, D. G. Austing, and S. Tarucha, Phys. Rev. B 65, 085325 (2002).

${ }^{28}$ M. Pi, M. Barranco, A. Emperador, E. Lipparini, and Ll. Serra, Phys. Rev. B 57, 14783 (1998). 